\title{
Single-mode inhibited-coupling fiber for sub-Doppler spectroscopy
}

\author{
T. Billotte ${ }^{1}$, G. Baclet ${ }^{3}$, J. H. Osório ${ }^{1}$, F. Delahaye ${ }^{2}$, V. Mançois ${ }^{3}$, A. Hilico ${ }^{3}$, M. Maurel ${ }^{1,2}$, M. Chafer ${ }^{1,2}$, F.

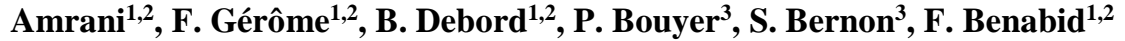 \\ ${ }^{I}$ GPPMM Group, Xlim research institute, University of Limoges, 123 avenue Albert Thomas 87060 Limoges, FRANCE \\ ${ }^{2}$ Glophotonics, 123 avenue Albert Thomas 87060 Limoges, FRANCE \\ ${ }^{3} L P 2 N$, Institut d'Optique d'Aquitaine, Rue François Mitterand 33400 Talence, FRANCE \\ Authore-mail address: f.benabid@xlim.fr
}

\begin{abstract}
We report on the use and qualification of the recently developed single-mode hybrid hollow-core photonic crystal fiber for a robust and long-life acetylene photonic micro-cell based optical frequency reference. () 2020 The Author(s)
\end{abstract}

The need for compact and stable frequency references is continuously increasing in several applications such as on-field sensing or high data-rate communication. Since its early development, hollow-core photonic crystal fiber (HCPCF) has been identified as a promising platform for coherent spectroscopy in gas-phase media thanks to the compactness and the long interaction length they provide. Indeed, once the fiber is filled with gas, thus forming what we call photonic micro-cell (PMC), sub-Doppler spectral signals can be generated via different means $[1,2]$ and laser frequency stabilization was demonstrated with different PMC configurations. Saturation absorption spectroscopy (SAS) in acetylene-filled HCPCF for telecom frequency reference is the most investigated [2-5]. Within this context, the best performances in acetylene-filled HCPCF achieved are summarized by an accuracy in ${ }^{12} \mathrm{C}_{2} \mathrm{H}_{2} \mathrm{P}(13)$ line optical frequency of $9.3 \mathrm{kHz}(1 \sigma$ error) using a $68 \mu \mathrm{m}$ diameter core Kagome HCPCF [2], and a fractional frequency instability (Allan deviation) of $4.10^{-12}$ at $1 \mathrm{~s}$ averaging time using the ${ }^{13} \mathrm{C}_{2} \mathrm{H}_{2} \mathrm{P}(16)$ line and employing a $8 \mu \mathrm{m}$ diameter core photonic bandgap HCPCF [4]. However, these results were obtained with fibers attached to a vacuum system. For portable and standalone PMC, the best reported results show a fractional frequency instability of $7.10^{-11}$ at $1 \mathrm{~s}$ integration time, and of $8.10^{-12}$ at $1 \mathrm{~s}$ when active polarization control is implemented [3]. There, the standalone PMC is formed by encapsulating the HCPCF tips with a borosilicate cell. Such a configuration suffers from low coupling efficiency ( $2.1 \mathrm{~dB}$ loss) and integration difficulty as it relies on free space optics. Furthermore, the long-term results show a strong and continuous increase with time in the SAS signal linewidth, indicating gas contamination due to the assembly process. The above shows that the performance of portable and sealed PMC-based optical frequency standards strongly and equally depends on both the cell gas loading and sealing process, and on the intrinsic physical and optical properties of the HCPCF. Indeed, an ideal PMC requires a gas loading and assembly process that ensure contamination-free, low insertion loss, insignificant etalon effects and ease-of-integration. Conversely, the HCPCF must combine stress resilient ultra-low loss, single-mode operation, polarization stability and large fiber core. The larger fiber core enables narrowing the transit-time limited SAS signal linewidth whilst the single modedness mitigates the spatial modal beating, which is a source of signal-to-noise ratio degradation, frequency instability and long term frequency drift [4]. With regard to the combination of robust single modedness and large core, a novel inhibitedcoupling guiding (IC) HCPCF has been reported to exhibit robust single-mode behavior in a core diameter of $\sim 35 \mu \mathrm{m}$ [6]. This IC-HCPCF is characterized by a hybrid cladding structure comprising in an inner ring of tubular amorphous lattice surrounded by a Kagome lattice (Fig. 1 I (b) 3).

Here, we report on an all-fiber, standalone and easy-integration PMC made with this newly developed IC-HCPCF. The PMC is assembled using a contaminant free end-capping technique [7]. The developed PMC shows ends equipped with FC/APC connectors for integration, robust single modedness, and fractional frequency instability of $3.10^{-11}$ at $1 \mathrm{~s}$ measured 642 days after its assembly.

Figure 1 summarizes the properties of the used hybrid cladding IC-HCPCF (Fig.1 I) and the acetylene PMC (Fig. $1 \mathrm{II}$ ). The used hybrid fiber exhibits a $4 \mathrm{~dB} / \mathrm{km}$ loss at the acetylene absorption region around $1550 \mathrm{~nm}$ (Fig. $1 \mathrm{I}$ (a)) and has a $35 \mu \mathrm{m}$ core diameter (Fig. 1 I (b) 3). In order to put the new fiber into perspective, the modal content of the hybrid IC-HPCF was measured using the S2 technique and compared with those of Kagome IC-HCPCF and Tubular IC-HCPCF with similar core size. The S2 measurement conditions were the same for the three fibers [e.g. fiber length (here 3 meters), fiber coiling radius...]. The hybrid fiber high order mode (HOM) content is clearly lower than the two other fibers, with $6 \%$ for the first lowest loss HOM (here LP11-like mode) and $2 \%$ ooo for the second lowest loss HOM (here LP31-like mode), which represent a power fraction over one order of magnitude lower than in the two other fibers. 


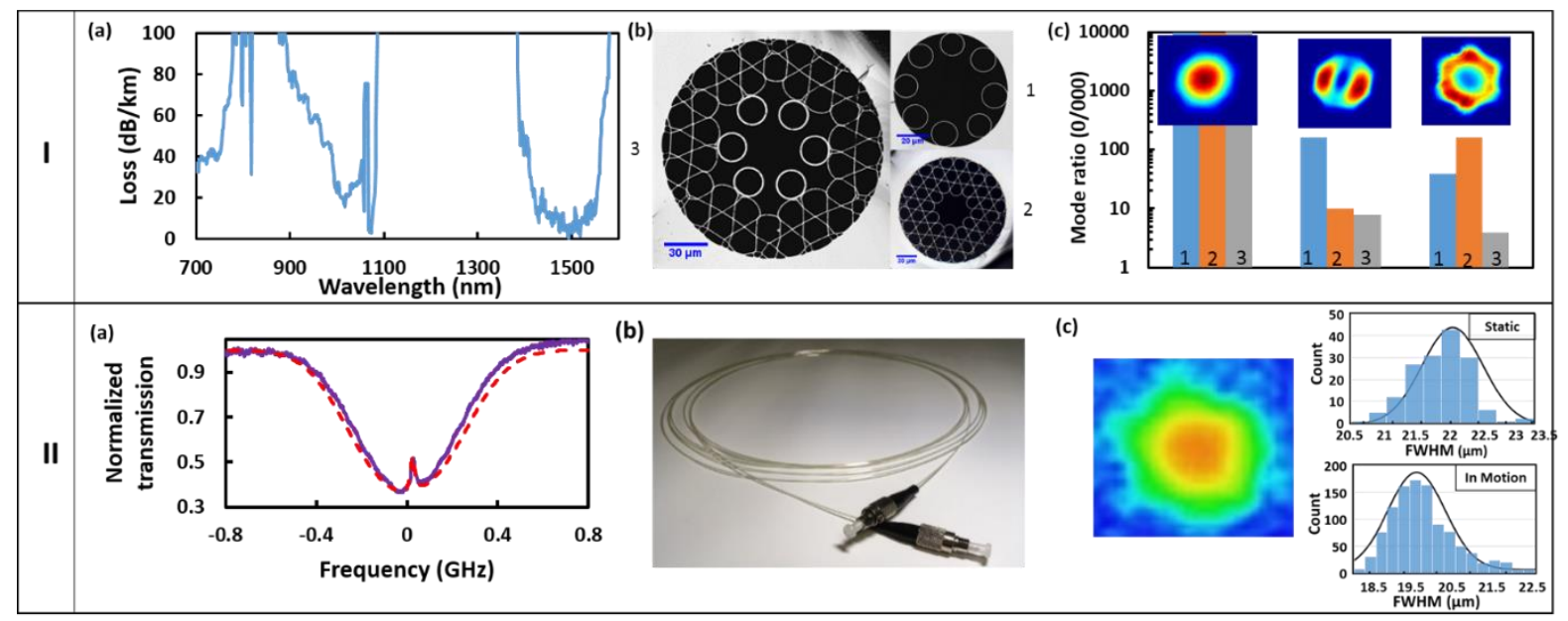

Fig. 1. (I)(a) Loss of the used hybrid fiber, (b)Microscope image of the compared fibers, (c) Modal content of the different fibers imaged in, (a) Measured by $\mathrm{S}^{2}$ technique. (II) (a) PMC SAS signal generated on P9 absorption line with $190 \mathrm{~mW}$ input pump beam power. (b) Picture of the final PMC (c) Near field intensity profile (left) and Full width at half maximum (FWHM) of this profile in static and motion.

Figure 1 II summarizes the properties of the PMC reported herein. The observed SAS signal in Fig. 1 II (a) is generated with a $190 \mathrm{~mW}$ input pump power entailing a contrast between absorption and transparency peak of 25\% and a FWHM of $17 \pm 3 \mathrm{MHz}$ which is, to the best of our knowledge, the actual narrower linewidth and higher contrast for a standalone PMC. Figure $1 \mathrm{II}$ (b) shows a photography of the $7 \mathrm{~m}$ long PMC with its FC/APC connectors. The total insertion loss was measured to $\sim 1.5 \mathrm{~dB}$, and is found to be the same for both direction. Furthermore the near field intensity profile (Fig.1 II (c)) is still single mode even under mechanical stress and vibration. Figure 1 II (c) shows the variation of the mode FWHM with and without stress of $\sim 2 \mu \mathrm{m}$ and the mode center position does not vary more than $0.6 \mu \mathrm{m}$.

Figure 2 shows the Allan variance of our PMC 642 days after its fabrication compared to free running laser and the work in [3] for plug\&play system. The laser is a $10 \mathrm{kHz}$ linewidth RIO diode with a free running Allan variance of around $7 \times 10^{-9} @ 10 \mathrm{~s}$ at a wavelength of $1533.461 \mathrm{~nm}$ corresponding to the P14 absorption line of acetylene. By locking it with the signal generated in our free space coupled PMC and comparing it with a Menlo frequency comb associated with a free running quartz $\left(2.10^{-12} @ 1 \mathrm{~s}\right)$, we obtain a fractional frequency instability of $4.10^{-11} @ 10^{3} \mathrm{~s}$ of integration time which is comparable with the one measured in [3], confirming the high performance and long life of our system.

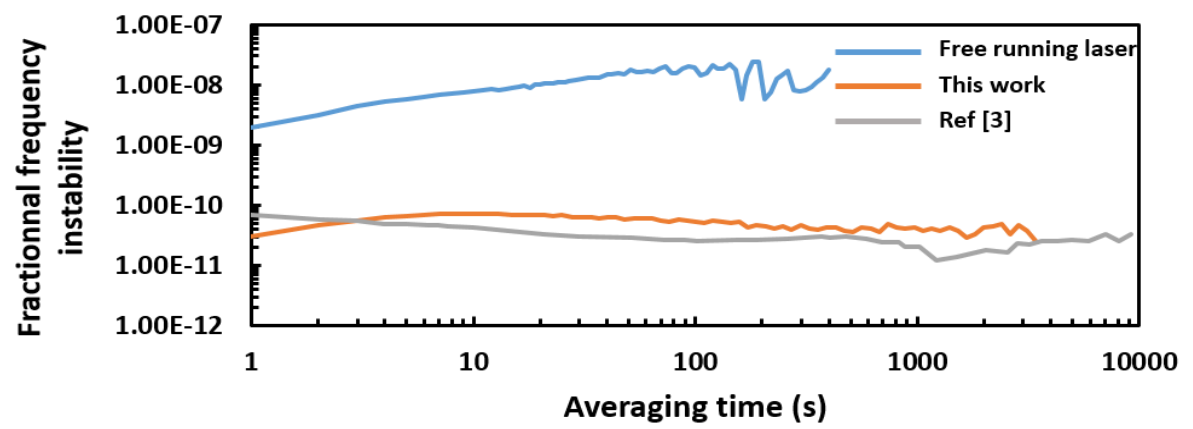

Fig. 2. Measured fractional frequency instability for free running laser, laser locked on P14 line with our PMC compared with

\section{References} a frequency comb, and the one measured in [3] for their plug\&play system.

1. $\quad$ F. Benabid, et al., Opt. Express 13, 5694 (2005).

2. K. Knabe, et al., Opt. Express 17, 16017 (2009).

3. M. Triches, et al., Appl. Phys. B Lasers Opt. 121, 251

(2015).

4. M. Triches, et al., Opt. Express 23, 11227 (2015)

5. C. Wang, et al., Appl. Opt. 52, 5430 (2013).

6. F. Amrani,et al., arXiv Prepr. arXiv2006.06375 (2020).
7. T. Billotte, et al., in CLEO Europe EQEC, OSA, ed. (2019).

Acknowledgements : CNES (R-S18/SU-0001-051, contract 3118), region Nouvelle Aquitaine (2016-1R60303-00007465, 20161R60303-00007466), AID (contract 2019650064), French National Research Agency (IdEx Bordeaux - LAPHIA ANR-10-IDEX-03-02) 